\title{
COMPARAÇÃO DAS PRÁTICAS AMBIENTAIS NOS HOTÉIS DA GALIZA E DO NORTE DE PORTUGAL
}

\section{THE COMPARISON OF ENVIRONMENTAL PRACTICES IN HOTELS LOCATED IN GALICIA AND NORTH OF PORTUGAL}

\section{María Dolores Sánchez-Fernández}

Doctora en la Universidad de A Coruña España, Facultad de Economía e Empresa.

Profesora e Investigadora Departamento de Análise Económica e ADE. Área Organización de Empresas. Miembro-Investigador de GREFIN, GEIDETUR en España, CICS

E-mail: msanchezf@udc.es

\section{Alfonso Vargas-Sánchez}

Profesor e Investigador Doctor, Catedrático de la Universidad de Huelva (España).

Coordinador del grupo de investigación GEIDETUR (Grupo de Investigación en Estrategias de Innovación y Desarrollo en la Empresa Turística)

E-mail: vargas@dem.uhu.es

\section{Paula Remoaldo}

Profesora e Investigadora Doctora, Asociada con Agregación de la Universidade do Minho (Portugal). Coordinadora del grupo de investigación "Space and Representations (SpaceR)" del Lab2pt (Laboratory of Landscape, Heritage and Territory) de la Universidade do Minho. Directora del programa de doctorado en Geografía de la Universidad do Minho E-mail: premoaldo@geografia.uminho.pt 


\section{COMPARAÇÃO DAS PRÁTICAS AMBIENTAIS NOS HOTÉIS DA GALIZA E DO NORTE DE PORTUGAL}

\section{RESUMO}

Na presente pesquisa pretendemos estudar a articulação da Teoria Institucional e as práticas ambientais nos hotéis de três, quatro e cinco estrelas, localizados na Galiza (Espanha) e no Norte de Portugal. Para o desenvolvimento desta pesquisa, pretendemos responder às seguintes perguntas: existe uma configuração institucionalizada em hotéis de três, quatro e cinco estrelas situados na Galiza e Norte de Portugal, que influencia o desenvolvimento de práticas ambientais? E quais dos mecanismos propostos pela Teoria Institucional exerce uma maior pressão sobre o desempenho ambiental na nossa unidade em estudo? Para esse efeito propomos quatro hipóteses e consideramos que a Teoria Institucional é uma abordagem válida que tenta explicar o comportamento das organizações. Como principal resultado desta investigação sobressai que a nossa unidade de estudo está incorporada num ambiente institucional claramente marcado por pressões coercitivas e normativas.

Palavras-chave: Teoria Institucional. Hotel. Legitimidade. Ambiente. Isomorfismo. 


\title{
THE COMPARISON OF ENVIRONMENTAL PRACTICES IN HOTELS LOCATED IN GALICIA AND NORTH OF PORTUGAL
}

\begin{abstract}
This objective of this investigation is to study the relationship between institutional theory and environmental practices in three, four and five-star hotels located in the North of Portugal and Galicia (Spain). To be able to carry out this research, the following questions were proposed: Is there an institutional framework in three, four and five-star hotels located in Galicia and Northern Portugal that influences the development of environmental practices? And which of the mechanisms proposed by institutional theory exerts greater pressure on environmental performance in our unit of study? With this in mind, four hypotheses are proposed and it is believed that institutional theory is a valid approach to explain the behavior in organizations. As a main result of this investigation it is important to highlight that our unit of study consists in an institutional environment clearly marked by the enforcement of coercive and normative pressures.
\end{abstract}

Keywords: Institutional Theory. Hotel. Legitimacy. Environment. Isomorphism. 


\section{INTRODUÇÃO}

O turismo continua a ser um dos motores econômicos de países como Espanha e Portugal, ainda que ambos os países estejam envolvidos, no momento de estudo, numa situação crítica em termos econômicos e sociais. Álvarez, Vila, Fraiz e Rio (2013) e Lopes (2010) em concordância com as estatísticas oficiais, reconhecem a importância do setor com destaque para o grande peso exercido na economia por empresas ligadas ao turismo. No entanto, este período marcado pela crise econômica é propício para vislumbrar o desempenho ambiental das empresas, uma vez que se trata dum período em que os cortes ocorrem em quase todos os recursos (. Perante o cenário atual torna-se necessário analisar de que forma os hotéis respondem à implementação de práticas ambientais.

Os postulados enquadrados na Teoria Institucional foram comparados e verificados, nos últimos anos, nas organizações da Administração Pública, tais como os municípios (Llamas-Sánchez, Garcia-Morales \& Martin-Tapia, 2013), as instituições de ensino superior (Dias, Martinago \& Gonçalves, 2006) e os hospitais (Bastidas \& Moreno, 2006). A prossecução do objetivo de legitimidade é mais necessária para a sobrevivência dessas entidades públicas do que para as privadas com fins lucrativos, pois, na maioria dos casos, não pode ser julgada com base na rentabilidade (Dobbin, et al., 1993, Casiles \& DavisBlake, 2002). No que diz respeito à esfera privada, Vargas-Sánchez e Riquel-Ligero (2016) centrou o seu estudo nos campos de golfe de Andaluzia, de Grobois (2016) no turismo de cruzeiros, Lavandoski, Vargas-Sánchez, Pinto e Silva (2016) no enoturismo e Restrepo e Rosero (2002) nas empresas colombianas e na sua internacionalização. Sobressaem também outros estudos, no setor financeiro na Colômbia (Murillo, González \& Rodríguez, 2010), na indústria de extração de azeite de oliva em Jaén (Moyano-Fuentes, 2001) ou no Rio Grande do Sul no que diz respeito aos aspectos ambientais (Arend \& Ferraz, 2005).

$\mathrm{Na}$ presente pesquisa analisamos o subsetor hoteleiro considerando a dimensão ambiental, que não tem recebido atenção suficiente em pesquisas anteriores realizadas à escala internacional.

A escolha do subsetor hoteleiro pretende dar consistência ao estudo, concretizando-se uma pesquisa comparativa entre duas regiões, a Galiza (em Espanha) e o Norte (em Portugal). Concentramo-nos nos hotéis de três, quatro e cinco estrelas, já que se acredita que podem ser realizadas práticas do foro ambiental com dimensão e organização Revista de Gestão e Secretariado-GeSec, São Paulo, v. 7, n. 2, p 191-212,mai./ago. 2016. 
suficiente. O estudo dessas empresas permite ter uma visão das organizações do setor privado e uma imagem mais precisa do processo de institucionalização e da formação dum ambiente institucionalizado.

A originalidade da investigação realizada deriva do referencial teórico usado (Teoria Institucional e práticas ambientais), do objeto de estudo e da área geográfica (hotéis de três, quatro e cinco estrelas situados na Galiza e no Norte de Portugal, que conjuntamente configuram uma Eurorregião), do contexto (crise económica), da área de estudo (esfera privada) e do tipo de organizações (lucrativas).

O principal objetivo deste estudo foi determinar se existe uma tendência de isomorfismo no desenvolvimento de práticas ambientais realizadas na nossa área de estudo. Acreditamos que a Teoria Institucional (DiMaggio \& Powell, 1991, Scott, 1995) é uma abordagem válida, que tenta explicar o comportamento das organizações isomórficas.

Os argumentos acima apresentados, juntamente com a relevância dos estudos sobre a Teoria Institucional e com a mudança institucional, levam-nos a considerar uma série de objetivos gerais a serem alcançados nesta pesquisa:

-Medir os determinantes que explicam a implementação de práticas ambientais e as variáveis do contexto institucional que afetam aos hotéis de três, quatro e cinco estrelas;

-Caracterizar o ambiente institucional em estes hotéis, localizados na Galiza e no Norte de Portugal, identificando as principais pressões existentes neste tipo de organizações.

$\mathrm{O}$ presente artigo divide-se em quatro itens. No primeiro item, é realizada uma revisão da literatura existente. Em seguida, na metodologia, apresenta-se o modelo de investigação usado, as etapas de investigação, as questões de partida e as hipóteses de trabalho. No terceiro item, são analisados os resultados depois de submetermos os dados aos vários testes. Por fim, apresentam-se as conclusões.

\section{REVISÃO DA LITERATURA}

A Teoria Institucional tem como principal base a legitimidade e os três pilares institucionais: coercitivo, normativo e cognitivo (Scott, 1995). De acordo com o institucionalismo regulador, as empresas legítimas são aquelas que operam tendo subjacentes as exigências legais. Os institucionalistas normativos interpretam a legitimidade das organizações adquiridas por obrigação moral. Este pilar é baseado na apropriação de normas e valores, através da acreditação do seu comportamento. Do ponto de vista cognitivo, a empresa Revista de Gestão e Secretariado -GeSec, São Paulo, v. 7, n. 2, p 191-212,mai./ago. 2016. 
geralmente adota um comportamento semelhante ao de outras organizações e adquire legitimidade no que diz respeito a tendências ou regras generalizadas. Isto é, age de acordo com o que é aceite no seu ambiente. Portanto, podemos concluir que os três pilares assumem uma abordagem diferente para adquirirem legitimidade. Cada um deles apresentam características peculiares e diferente dos outros. Estas características envolvem uma ênfase diferente com base no compromisso de alcançar a lógica, os mecanismos e os indicadores apresentados. Por outro lado, as implicações de cada um dos pilares institucionais são definidas de acordo com a rotina, as estruturas sociais e a cultura que cada um adota.

A comunidade científica defende que a legitimidade se tornou um dos elementos críticos para as organizações (Castelló \& Lozano, 2011), nomeadamente a nível mundial (Kostova \& Zaheer, 1999). Vários autores (Scott, 1995; Suchman, 1995; Deephouse, 1996; Ruef \& Scott, 1998; Kostova \& Zaheer, 1999; Kostova \& Roth, 2002; Golant \& Sillince, 2007) identificam os diferentes tipos de legitimidade devido ao interesse das empresas em encontrarem novas formas de legitimidade (Palazzo \& Scherer, 2006), e almejarem expandir a pesquisa para a esfera política como por ejemplo os agentes estatais - autarquias, etc (Walsh, Weber \& Margolis, 2003; Scherer \& Palazzo, 2007).

Durante a última década, vários trabalhos foram desenvolvidos considerando a legitimidade das organizações (Baum \& Oliver, 1991; Suchman, 1995; Deephouse 1996; Hunt \& Aldrich, 1996; Ruef \& Scott, 1998; Egels-Zanden \& Wahlqvist, 2007; Díez, Blanco \& Prado, 2010; Llamas-Sánchez, Garcia-Morales \& Martin-Tapia, 2013; Vargas-Sánchez \& Riquel-Ligero, 2016). Esses autores sugerem que a legitimidade é um fator chave para sobreviver e fazer crescer as organizações. Díez, Blanco e Prado (2010) referem que se podem realizar práticas para gerenciar e determinar a legitimidade da organização e a gestão da legitimidade pode realizar-se por diferentes vias: aumentar, manter ou recuperar.

Com base no exposto, podemos encontrar a relação entre a capacidade das empresas para tratarem a legitimidade e obterem recursos. Díez, Blanco e Prado (2010) relacionam o nível de legitimidade alcançada pela empresa e a obtenção de maior ou menor benefício. As empresas que atingem um nível mais elevado de legitimidade tendem a conseguir uma posição mais favorável para alcançarem lucros mais elevados. Egels-Zanden e Wahlqvist (2007) destacam que as empresas estão interessadas em serem identificadas como empresas responsáveis, com o objetivo estratégico de aumentar ou remediar a situação da percepção de legitimidade das principais partes interessadas: o cliente e o consumidor.

Revista de Gestão e Secretariado -GeSec, São Paulo, v. 7, n. 2, p 191-212,mai./ago. 2016. 
A legitimidade pode ser gerenciada de acordo com o objeto de desejo das organizações, como seja, a vantagem competitiva ou o apoio dos grupos de interesse. É gratuita e permite que as empresas ganhem mais recursos críticos (Díez, Blanco \& Prado, 2010), favorecendo o seu crescimento ou permitindo a sua sobrevivência. Possibilita também um bom relacionamento tanto com os clientes como com os consumidores.

A legitimidade é frequentemente calculada pelos gestores (Castelló \& Lozano, 2011). As empresas desenvolvem respostas estratégicas às instituições, o que implica, de forma proactiva, o preenchimento de lacunas institucionais. No entanto, as empresas apenas refletem formas institucionalizadas de comportamento (Oliver, 1991).

As organizações têm estratégias para ganhar, manter ou recuperar a legitimidade (Suchman, 1995). Além de estratégias existem grupos capazes de conferir legitimidade como, por exemplo, as organizações governamentais e não-governamentais e os sindicatos (Meyer \& Scott, 1983; Galaskiewicz, 1985; Baum \& Oliver, 1991; Meyer \& Rowan, 1997; Piazza, 2002; Braun \& Gearhart, 2004; Egels-Zanden \& Kallifatides, 2006).

Autores relevantes que estudam o paradigma da Teoria Institucional concentramse na questão ambiental (Rivera, 2004; Shah \& Rivera, 2007; Riquel, 2010; Zhu, Sarkis \& Lai, 2011; Vargas-Sánchez \& Riquel-Ligero, 2012; Zapata \& Hall, 2012; Shah \& Rivera, 2013; Llamas-Sánchez, Garcia-Morales \& Martin-Tapia, 2013; Vargas-Sánchez \& RiquelLigero, 2016).

Num periodo crise econômica é pertinente vincularem-se as questões ambientais, pois podem afetar outros interesses, como a sobrevivência da organização ou a obtenção de benefícios imediatos em relação a outro tipo de problemas. Mesmo as organizações podem adotar um comportamento ambientalmente irresponsável para atingir os objetivos.

A Teoria Institucional tem uma origem multidisciplinar (Perdomo \& Escobar, 2011) e, por isso, encontra muitas aplicações em vários ramos da ciência. Nos últimos anos, abriu o campo para a orientação da administração de empresas (Gerde \& Wokutch,1998; Rowley \& Berman, 2000; De Bakker, Groenewegen \& Den Hond, 2005; Lockett, Moon \& Visser, 2006; Bigné et al., 2010). Nesta pesquisa queremos contribuir para o setor de organização de negócios, com novos contributos e avanços no contexto da Teoria Institucional. 


\section{Hipóteses}

Com base na revisão da literatura sugerem quatro hipóteses:

H1. A pressão coercitiva produzida por leis e outras regulamentações aplicáveis às atividades da organização influenciam positivamente a adoção de práticas ambientais.

H2. A aceitação de valores e normas que derivam das pressões regulatórias que ocorrem no contexto das organizações influenciam positivamente a adoção de práticas ambientais.

H3. A imitação de práticas ambientais percebidas como organizações de sucesso influenciam positivamente a adoção de práticas ambientais.

H4. A adoção de práticas ambientais influenciam positivamente em a legitimidade social procurada pelas organizações.

\section{MÉTODO}

\section{Procedimentos de Coleta de Dados}

Tendo em conta os objetivos delineados, foi desenvolvido um estudo quantitativo. Com esta finalidade foi elaborado um questionário com base nas caraterísticas do objeto de estudo (os hotéis) e dos informantes (diretores de hotel). O questionário foi redigido na língua oficial de cada país (Espanhol e Português), segundo as recomendações de Vitell, Ramos e Nishihara (2010).

O resultado foi um questionário composto de 75 itens divididos em nove seções. Em grande parte das questões usou-se uma escala de Likert de 5 níveis, variando desde a opção "discordo completamente" até à opção "concordo completamente". As três primeiras seções são de escalas já validadas (escala de Likert de 5 níveis): a escala contexto institucional (Kostova \& Roth, 2002; Vargas-Sánchez \& Riquel-Ligero, 2012; LlamasSánchez, Garcia-Morales \& Martin-Tapia, 2013), a escala que contem o construto "práticas ambientais" (Gallardo-Vázquez \& Sánchez-Hernández, 2012) e a escala legitimidade (Deephouse, 1996; Fernández, 2001; Vargas-Sánchez \& Riquel-Ligero, 2012; LlamasSánchez, Garcia-Morales \& Martin-Tapia, 2013). As seções que recolhem as caraterísticas sociodemográficas, de classificação e de categorização incluem as principais variáveis consideradas nos relatórios elaborados pelo Instituto Nacional de Estatística de Espanha e de 
Portugal, pelo Instituto de Estudos de Turismo de Espanha, pela Conta Satélite de Turismo de Espanha e de Portugal e pelo Instituto Galego de Estatística.

Além disso, o questionário foi revisto por três especialistas nas áreas de gestão, geografia e turismo. Na sequência dos comentários acadêmicos aplicou-se o pré-teste a dez gerentes de hotéis diferentes em cada uma das regiões ( 5 em Galiza e 5 em Portugal) durante o mês de abril de 2012 .

Foram introduzidas algumas alterações no questionário com base nas sugestões recolhidas durante a realização do pré-teste. A aplicação do questionário foi efetivada entre maio e agosto de 2012. Para tal, foram contactados semanalmente os diretores de hotel por email, correio postal, telefone, fax ou efetuando visitas ao local.

\section{Amostra}

O erro de amostragem obtido no caso da Região Norte de Portugal é de 6,74\% e de 6,56\% no caso da Galiza, onde $\mathrm{p}=\mathrm{q}=0,5$. Os questionários usados na presente análise correspondem ao número de questionários válidos, que no caso do Norte de Portugal se cifraram em 44 e no caso da Galiza em 65. Estes dados representam uma taxa de respostas de $29,93 \%$ e de $30,80 \%$, respectivamente. As percentagens da amostra foram consideradas adequadas. Estabelecemos como nível mais exigente o correspondente ao estudo de VargasSánchez e Riquel-Ligero (2012), com uma taxa de respostas de 33,3\%, bastante acima da taxa obtida por Llamas-Sánchez, Garcia-Morales e Martin-Tapia (2013) com 21,33\%, por Gallardo-Vázquez e Sánchez-Hernández (2012) com 11,07\% e por Godos e Fernández (2011), com 5\%.

\section{Procedemento de Análise}

Foi utilizado o SPSS (versão 18.0), o pacote Microsoft Office (versão 2003) e o software Visual-PLS para aplicar a metodologia de equações estruturais. A análise empírica encerrou em duas fases:

Na primeira fase desenvolvemos os testes de unidimensionalidade de escalas com o objetivo de realizar análise fatorial para os construtos que compõem o modelo de pesquisa. A aplicação desta metodologia permitiu reduzir a informação contida num conjunto de variáveis originais passando a usar-se um conjunto menor com a perda mínima de informação Revista de Gestão e Secretariado -GeSec, São Paulo, v. 7, n. 2, p 191-212,mai./ago. 2016. 
(Bartlett, 1951; Kaiser, 1970; Nunnally, 1978; Wubneh, 1987; Verdu, 2002; Aymerich \& Meseguer, 2004; Perez, 2005; Wang, 2005).

Usamos no seguinte passo o modelo de pesquisa baseado na Técnica de Mínimos Quadrados Parciais (PLS) que se baseia na metodologia de Equações de Modelos Estruturais (SEM), e que exige a realização dum modelo de medição e um modelo estrutural.

Numa segunda fase desenvolvemos os modelos de medição, especificando as relações entre as variáveis observadas ou indicadores com as variáveis latentes ou construtos. Em seguida, desenvolvemos o modelo estrutural no qual se avalia a existência e a magnitude da relação entre variáveis latentes ou construtos (Barclay, Higgins \& Thompson, 1995).

Para o desenvolvimento desta pesquisa, pretendemos responder às seguintes perguntas: existe uma configuração institucionalizada em hotéis de três, quatro e cinco estrelas situados na Galiza e Norte de Portugal, que influencia o desenvolvimento de práticas ambientais? E quais dos mecanismos propostos pela Teoria Institucional exerce uma maior pressão sobre o desempenho ambiental na nossa unidade em estudo? Para esse efeito propomos três hipóteses (H1 até H3).

Propomos também responder à seguinte questão: existe uma relação direta positiva entre as práticas ambientais e a legitimidade nos hotéis em estudo? Para isso, colocamos a seguinte hipótese 4.

\section{O Modelo}

A partir da revisão da literatura, propusemos o seguinte modelo estrutural que se relaciona com as pressões do contexto institucional (PO, PN, PM) para a dimensão ambiental da RSC (PRMA), e estas prácticas ambientais (PRMA) com a legitimidade (LG). As hipóteses correspondem às relações entre os construtos do nosso modelo de pesquisa, como é salientado na Figura 1.

\section{Figura 1- Modelo de pesquisa proposto}

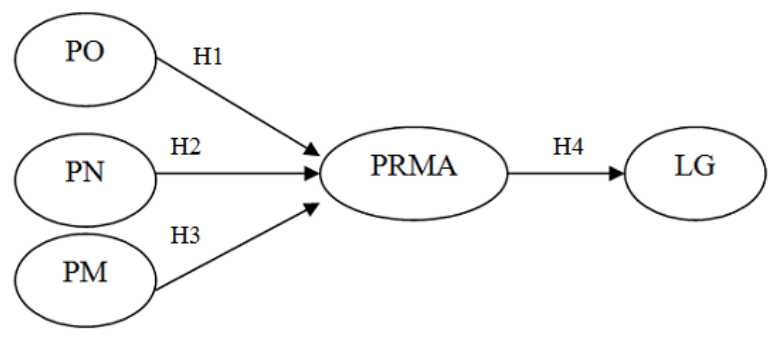

Fonte: Elaboração própria. 
Os construtos PRMA e LG compõem-se, cada um, de dois construtos de primeira ordem. Sendo que PRMA é composto de PMP e PMA e LG de LO e LI. O modelo contém sete construtos (ou variáveis latentes) de primeira ordem refletivos (PO, PM, PN, PMP, PMA, LI e LO) e dois construtos de segunda ordem formativos (PRM e LG), em ambos os modelos (Galiza / Norte de Portugal). Considerou-se um total de 23 indicadores (ou variáveis observáveis) na Galiza, e em Portugal 25 indicadores de relações de tipo refletivas. Este conjunto de variáveis observáveis é obtido depois de testar a unidimensionalidade da escala, isto é, os itens que permanecem são aqueles que agregam valor para os fatores (construtos).

Para a medição das diferentes variáveis, utilizamos um questionário estruturado, descrito anteriormente. De seguida, são apresentadas as variáveis latentes do modelo e os indicadores que compõem os construtos de primeira e segunda ordem. Os construtos de primeira ordem com indicadores refletivos são:

- Pressão coercitiva (PO) - conhecimento das leis (A1), a aplicação da lei (A2), reguladores (A3), existência de acordos (A4);

- Pressão normativa (PN) - obrigação moral (A5), concordância com os valores ambientais (A6) e as normas sociais (A7);

- Pressão mimética (PM) - o conhecimento de experiências (A8), modelos (A9), imitação de práticas (A10) e o conhecimento do bem sucedido (A11);

- Práticas ambientais predisponentes (PMP) - minimizar o impacto ambiental (C13) e o uso de produtos com baixo impacto ambiental (C14);

- Práticas ambientais de ação (PMA) - de economia de energia (C15), de fontes alternativas de energia (C16), redução de impacto de planejamento de investimentos (C17), de redução de emissões (C18), itens orgânicos (C19), embalagens recicláveis (C20);

- A legitimidade da organização (LO) - o reconhecimento social (A12) e os valores organizacionais (A13);

- Legitimidade relativamente às partes interessadas (LI) - Administração Pública (B1), empregados (B2), cidadãos (B3), meios de comunicação (B4), clientes (B5), fornecedores (B6), associações (B7), indústria de negócio (B8) e as relações com outros grupos de pressão (B9).

Os construtos de segunda ordem formativos são:

- Legitimidade (LG) - a legitimidade da organização (LO) e a legitimidade relativamente às partes interessadas $(\mathrm{LI})$; 
- Práticas Ambientais (PRMA) - Práticas Ambientais Predisponentes (PMP) e Práticas Ambientais de Ação (PMA).

\section{RESULTADOS}

Nos primeiros testes realizados de unidimensionalidade, dos 29 itens iniciais, no modelo da Galiza permaneceram 23 itens e em Portugal 25 (ver Tabela 1). As outras variáveis foram eliminadas porque não satisfaziam os requisitos estabelecidos (teóricos/estatísticos). Ambos os modelos possuem o mesmo número de construtos (ver Figura 1). Os construtos PM, PMA e LI não têm o mesmo número de itens, enquanto os restantes construtos (PO, PN, PMP e LO) têm o mesmo número. Após realizar o teste de unidimensionalidade, incluimos os construtos formados no modelo de aplicação e realizamos a análise dos modelos de medida e a análise estrutural. Para a construção dos construtos baseamo-nos na diferença de indicadores refletivos e formativos propostos por Sarabia et al. (1999).

\section{Análise dos Modelos de Medida}

$\mathrm{Na}$ análise dos modelos de medição submetemos todos os construtos refletivos aos vários testes, exceto os formativos (PRMA e LG). Consideramos que as medidas dum construto, no caso de construtos formativos, não precisam de ser correlacionados e não se aplicam medidas de consistência interna como o Alfa de Cronbach, a fiabilidade composta e o AVE (Sarabia et al., 1999).

Avaliamos a confiabilidade dos itens individuais, ou seja, analisamos as cargas ou correlações dos diferentes indicadores, com os seus respectivos construtos. O nível de aceitação, que definimos, é fornecido por Falk e Miller (1992), segundo o qual as cargas devem ser maiores ou iguais a 0,505. No nosso caso, todas as cargas das variáveis nos diferentes modelos cumprem os critérios estabelecidos, não excluindo nenhuma (ver Tabela 1).

Para avaliar a confiabilidade do construto (pc) seguimos a perspetiva de Calvo de Mora e Criado (2005) e Nunally (1978) que afirmam que temos um nível suficiente de confiança acima de 0,7 (pc) para estados iniciais de pesquisa, ou seja, invesgigações não muito experimentadas. No nosso caso, excedemos a barreira de 0,8 . Na Tabela 1 indicamos os valores obtidos a partir da fiabilidade composta (pc) dos construtos refletivos que compõem o Revista de Gestão e Secretariado-GeSec, São Paulo, v. 7, n. 2, p 191-212,mai./ago. 2016. 
modelo proposto para a investigação. Os construtos formativos não foram submetidos a este teste baseando-nos em Sarabia et al. (1999).

A validade convergente (AVE) foi observada em todos os casos. Os valores são maiores do que 0,5 , isto é, garantimos que mais de $50 \%$ da variância variação dos construtos é devido aos seus indicadores (Fornell \& Lacker, 1981). A Tabela 1 mostra os valores da variância média obtida no modelo de pesquisa proposto. Temos comprovado que o AVE é maior que as correlações ao quadrado entre este construto e os outros que compõem o modelo de investigação (Fornell e Lacker, 1981). Todos cumpriram a condição e, por esse motivo, podemos afirmar que os construtos têm a propriedade da validade discriminante.

$\mathrm{Na}$ análise dos modelos de mensuração, o modelo do Norte de Portugal tem uma maior robustez, confiabilidade e validade, do que o modelo da Galiza.

Descrevemos na Tabela 1 os resultados da análise dos modelos de medição.

Tabela 1: Avaliação de modelos de medição

\begin{tabular}{|c|c|c|c|}
\hline Teste & Construtos & Norte de Portugal & Galiza \\
\hline \multirow{6}{*}{ Cargas } & PO & $\begin{array}{l}\text { A1: } 00,80 \\
\text { A2: } 0,80 \\
\text { A3: } 0,91 \\
\text { A4: } 0,93\end{array}$ & $\begin{array}{l}\text { A1: } 0,73 \\
\text { A2: } 0,76 \\
\text { A3: } 0,73 \\
\text { A4: } 0,72\end{array}$ \\
\hline & PN & $\begin{array}{l}\text { A5: } 0,65 \\
\text { A6: } 0,85 \\
\text { A7: } 0,86\end{array}$ & $\begin{array}{l}\text { A5: } 0,89 \\
\text { A6: } 0,91 \\
\text { A7: } 0,59\end{array}$ \\
\hline & PM & $\begin{array}{l}\text { A8: } 0,96 \\
\text { A9: } 0,94 \\
\text { A10: } 0,62 \\
\text { A11: - }\end{array}$ & $\begin{array}{l}\text { A8: } 0,81 \\
\text { A9: } 0,86 \\
\text { A10: } 0,87 \\
\text { A11: } 0,64\end{array}$ \\
\hline & PMA & $\begin{array}{l}\text { C15: - } \\
\text { C16: - } \\
\text { C17: } 0,83 \\
\text { C18: } 0,90 \\
\text { C19: } 0,81 \\
\text { C20: } 0,92\end{array}$ & $\begin{array}{l}\text { C15: } 0,67 \\
\text { C16: - } \\
\text { C17: } 0,71 \\
\text { C18: } 0,87 \\
\text { C19: - } \\
\text { C20: } 0,71\end{array}$ \\
\hline & LO & $\begin{array}{l}\text { A12: } 0,88 \\
\text { A13: } 0,88\end{array}$ & $\begin{array}{l}\text { A12: } 0,82 \\
\text { A13: } 0,82\end{array}$ \\
\hline & LI & $\begin{array}{l}\text { B1: } 0,83 \\
\text { B2: } 0,82 \\
\text { B3: } 0,82 \\
\text { B4: } 0,76 \\
\text { B5: } 0,82 \\
\text { B6: } 0,86 \\
\text { B7: } 0,56 \\
\text { B8: } 0,84 \\
\text { B9: }-\end{array}$ & $\begin{array}{l}\text { B1: - } \\
\text { B2: } 0,84 \\
\text { B3: - } \\
\text { B4: - } \\
\text { B5: } 0,79 \\
\text { B6: - } \\
\text { B7: } 0,66 \\
\text { B8: }- \\
\text { B9: } 0,89\end{array}$ \\
\hline \multicolumn{2}{|c|}{ AVE } & $\begin{array}{l}\text { PO: } 0,74 \\
\text { PN: } 0,62 \\
\text { PM: } 0,73 \\
\text { PMP: } 0,73\end{array}$ & $\begin{array}{l}\text { PO: } 0,54 \\
\text { PN: } 0,65 \\
\text { PM: } 0,64 \\
\text { PMP: } 0,77\end{array}$ \\
\hline
\end{tabular}

Revista de Gestão e Secretariado -GeSec, São Paulo, v. 7, n. 2, p 191-212,mai./ago. 2016. 


\begin{tabular}{|l|l|l|}
\hline & PMA: 0,75 & PMA: 0,55 \\
& LO: 0,78 & LO: 0,67 \\
& LI: 0,63 & LI: 0,64 \\
\hline & PO: 0,92 & PO: 0,82 \\
& PN: 0,83 & PN: 0,85 \\
& PM: 0,89 & PM: 0,87 \\
& PMP: 0,85 & PMP: 0,87 \\
& PMA: 0,92 & PMA: 0,83 \\
& LO: 0,88 & LO: 0,89 \\
& PO: 0,92 & PO: 0,82 \\
& PN: 0,83 & PN: 0,85 \\
& PM: 0,89 & PM: 0,87 \\
& PMP: 0,85 & PMP: 0,87 \\
& PMA: 0,92 & PMA: 0,83 \\
PC: & LO, & LO: 0,89 \\
Ĺreas geográficas: NP: Norte de Portugal; G: Galiza. 0,88 \\
Construtos reflectivos: PO: pressão coercitiva, PN: pressão regulatória, PM: pressão mimética; PMP: práticas \\
ambientais predisponentes; PMA: práticas ambientais de ação; LO: legitimidade da organização, LI: \\
legitimidade respeito as partes interessadas. \\
Construtos formativos: PMRA: práticas ambientais; LG: legitimidade. \\
-: Não contêm a variável. \\
\hline
\end{tabular}

Fonte: Elaboração própria.

\section{Análise do Modelo Estrutural}

Nesta seção, vamos tentar determinar o poder preditivo do modelo de pesquisa. Analisamos o poder preditivo do modelo usando o valor da variância explicada $\left(\mathrm{R}^{2}\right)$, para as variáveis latentes dependentes (Falk \& Miller, 1992). Segundo Falk e Miller (1992) temos que usar valores iguais ou superiores a 0,1 como também os valores adequados de variância explicada. Devemos notar que, da mesma forma, os valores menores são estatisticamente significativos, embora indicando um baixo nível de variável latente preditiva. Os resultados obtidos são os seguintes R²: PRMA: 0,18(G)/0,40(NP); LG: 0,12(G)/0,27(NP).

$\mathrm{Na}$ análise do poder preditivo do modelo proposto, baseado no $\mathrm{R}^{2}$, ambos os casos são estatisticamente significativos. O modelo que tem o maior poder preditivo é do Norte de Portugal.

$\mathrm{Na}$ análise estrutural estimamos que os coeficientes $\beta$, também chamados coeficientes path deverão ser iguais ou exceder 0,2 segundo Chin (1998), Roldán (2000) e Riquel (2010). Os resultados dos coeficientes $\beta$ são incorporados a seguir: PÓ $\rightarrow$ PRMA: 0,21(G)/0,49(NP); PN $\rightarrow$ PRMA: $\quad 0,30(\mathrm{G}) / 0,20(\mathrm{NP}) ; \quad$ PM $\rightarrow$ PRMA: $\quad 0,19(\mathrm{G}) / 0,09(\mathrm{NP})$, PRMA $\rightarrow$ LG: $0,21(\mathrm{G}) / 0,52(\mathrm{NP})$. 
$\mathrm{Na}$ análise do modelo estrutural, os coeficientes $\beta$ obtidos a partir da relação entre as práticas ambientais e a legitimidade é alta e positiva. Ambos os modelos satisfazem as relações com o contexto institucional normativo e coercitivo, mas não com o mimético. A relação entre os construtos PRMA e LG adquire um valor significativo nos dois modelos. A pressão que exerce maior influência, no caso da Galiza, é a normativa, e no caso do Norte de Portugal é o contrário, a pressão coercitiva é mais intensa que a normativa. Em ambos os casos, a pressão mimética não é significativa.

$\mathrm{Na}$ análise da força das hipóteses e o seu contraste, nota-se que genericamente as hipóteses são aceites, exceto na relação entre a pressão mimética e as práticas ambientais. Paralelamente, o modelo do Norte de Portugal apresenta os melhores parâmetros.

No teste de hipótese baseado no modelo especificado, conclui-se que três das quatro hipóteses são aceites (H1, H2 e H4). Além disso, o modelo do Norte de Portugal revela os melhores resultados estatísticos, os maiores atributos de validade e de confiabilidade, e recebe um poder preditivo elevado.

\section{CONCLUSÕES}

O elemento-chave da Teoria Institucional é o contexto em que as empresas estão inseridas e a legitimidade é a meta organizacional para garantir a sua sobrevivência. Como foi mencionado no início deste artigo, o isomorfismo institucional é baseado em três mecanismos: o coercitivo, o normativo e o mimético, que leva à homogeneização das organizações na busca de aceitação social e garantia de obtenção de recursos de sobrevivência.

A legitimidade é um fator chave para as organizações sobreviverem e crescerem (Suchman, 1995; Deephouse, 1996; Hunt \& Aldrich, 1996; Ruef \& Scott, 1998; Díez, Blanco \& Prado, 2010) e é uma peça essencial para aceder a recursos críticos que afetam o crescimento e a sobrevivência da organização (Baum \& Oliver, 1991 e Díez, Blanco \& Prado, 2010). Neste domínio obtivemos resultados no nosso estudo que apoiam a nossa hipótese inicial. A legitimidade é importante porque a sua deterioração ou a sua perda pode levar à falência da empresa (Ahlstrorn \& Bruton, 2001; Chen, Griffith \& Hu, 2006; Díez, Blanco \& Prado, 2010). As instalações e atividades no setor do turismo têm um impacto direto sobre as práticas ambientais tais como, espaços abertos e os locais estratégicos. Estas são atividades 
que afetam o movimento de pessoas que influenciam o ambiente, a paisagem, a vida diária da comunidade local, as empresas e os cidadãos.

À medida que estas instalações crescem há mais preocupação de aplicar práticas ambientais.

Na configuração das pressões que moldam o ambiente institucional, podemos observar que a pressão coercitiva, que é, a existência de orgãos reguladores que promovem programas e aplicam práticas de gestão ambiental, é o principal indicador que forma este tipo de pressão, no caso dos hotéis localizados no Norte de Portugal. Este resultado está em consonância com as conclusões apresentadas em obras como Kostova e Roth (2002), Riquel (2010) e Llamas-Sánchez, Garcia-Morales e Martin-Tapia (2013). Da mesma forma, no que diz respeito à pressão normativa observamos que o maior contributo é a meta a alcançar, sobretudo nos hotéis localizados na Galiza, em tentar conseguir uma coerência nos valores sociais do ambiente e pôr em funcionamento este tipo de política ambiental, reafirmando os postulados defendidos por Jennings e Zandbergen (1995) e Voss, Cable e Giraud (2000). Finalmente, deparamo-nos com a pressão mimética que não exerce influência institucional, encontrada noutros estudos bem sucedidos no campo de ação, de acordo com DiMaggio e Powell (1991) e Deephouse (1996).

Neste último caso, a teoria não corresponde à realidade, ou seja, os diretores de hotéis de três, quatro e cinco estrelas localizados na Galiza e no Norte de Portugal não consideram a pressão mimética. Esses gerentes não têm em consideração as práticas ambientais de outros hotéis bem sucedidas. Eles não são da opinião que mostram resultados em termos de rentabilidade a curto prazo. A gestão está, deste modo, preocupada com o imediatismo e não com o longo prazo, o que não coincide com a linha a seguir para a correta aplicação destas práticas numa organização.

Nos resultados do nosso estudo empírico, pudemos ver como a legitimidade e o apoio social afeta o comportamento na nossa unidade de estudo, destacando a necessidade de apoio social que tem este tipo de organizações tanto na Galiza como no Norte de Portugal. Na variável legitimidade observa-se que o que implica maior carga é manter relações estáveis com as partes interessadas em geral e, particularmente, com funcionários e clientes.

Em resumo, podemos afirmar que os hotéis de três, quatro e cinco estrelas são incorporados num ambiente institucional claramente marcado por pressões coercitivas e normativas, ignorando a pressão mimética, confirmando que não se realizam práticas isomórficas no meio ambiente em diferentes territórios.

Revista de Gestão e Secretariado -GeSec, São Paulo, v. 7, n. 2, p 191-212,mai./ago. 2016. 
Esta pesquisa apresenta uma série de limitações. Primeiro, é de salientar que nos construtos apelidados de PM, PMA e LI, de ambos os modelos, não foi usado o mesmo conjunto de variáveis. Outra limitação está relacionada com o tamanho da amostra, não podendo generalizar os resultados para todo o país (Espanha e Portugal). Portanto, este estudo é replicável para futuras pesquisas em áreas geográficas de maior dimensão, que podem incluir uma comparação entre diferentes setores. Outra limitação também está relacionada com a recolha da informação, já que o nosso estudo contou com medidas baseadas nas percepções dum único informante, ou seja, os gerentes de hotel. Assim, pesquisas futuras devem procurar os pontos de vista de outras partes interessadas na linha proposta por Kumar, Stern e Anderson (2003). Além disso, seria prudente a aplicação doutras técnicas de confirmação, podendo esta ser uma proposta de futura linha de pesquisa, em que se aumente o volume da amostra e se propõe a criação duma análise de grupos múltiplos. Resumindo, a maioria das limitações deste estudo devem ser encaradas como oportunidades para pesquisas futuras.

\section{REFERÊNCIAS}

Ahlstrom, D. \& Bruton, G.D. (2001). Learning from successful local private firms in China: Establishing legitimacy. Academy of Management Executive, 15(4), 72-83.

Alvarez García, J., Vila Alonso, M., Fraiz Brea, J. A., \& Río Rama, M. C. (2013). Análisis de las relaciones de dependencia entre los factores críticos de la calidad y los resultados. Sector de alojamiento turístico en España. Investigaciones Europeas de Dirección y Economía de la Empresa, 19(2), 74-89. doi:10.1016/j.iedee.2012.08.001

Arend, Marcelo \& Ferraz Cário, Silvio Antonio (2005) Origens e determinantes dos desequilíbrios no Rio Grande do Sul: uma análise a partir da Teoria Institucional de Douglas North. Ensaios FEE, 26, 63-94.

Aymerich Martínez, J. \& Meseguer Artola, A. (2004). Investigación descriptiva: análisis de información. En Meseguer Artola, A. \& Vilaseca Requena, J (Coord). Estadística aplicada. Catalunya: Fundació Universitat Oberta de Catalunya.

Barclay, D, Higgins, C. \& Thompson, R. (1995). The Partial Least Square (PLS) Approach to causal modelling: Personal computer adoption and use as an illustration. Technology studies, special issue on research methodology, 2(2), 285-309.

Bartlett, M.S. (1951). A further note on tests of significance in factor analysis. British Journal of Psychology, 4, 1-2.

Revista de Gestão e Secretariado-GeSec, São Paulo, v. 7, n. 2, p 191-212,mai./ago. 2016. 
Bastidas Bermúdez, E.L. \& Moreno Freitez, Z. (2006). El cuadro de mando integral en la gestión de las organizaciones del sector público: El caso Universidad Centroccidental Lisandro Alvarado. Revista Universo Contábil, 2(3), 104-118.

Baum, J.A. \& Oliver, C. (1991). Institutional linkages and organizational mortality. Administrative Science Quarterly, 36(2), 187-219.

Bigné, E., Alvarado, A., Currás, R. \& Rivera, J. (2010). Latest evolution of academic research in corporate social responsibility: An empirical análisis. Social Responsibility Journal, 6, 332-344.

Braun, R. \& Gearhart, J. (2004). Who should code your conduct? Trade union and NGO differences in the fight for workers rights. Development in Practice, 14(1-2), 183- 196.

Calvo de Mora, A. \& Criado, F. (2005). Análisis de la validez del modelo europeo de excelencia para la gestión de la calidad en instituciones universitarias: un enfoque directivo. Revista Europea de Dirección y Economía de la Empresa, 3(14), 41-58.

Casiles, M. \& Davis-Blake, A. (2002). When accreditation standards change: Factors affecting differential responsiveness of public and private organizations. Academy Management Journal, 1(45), 180-195.

Castelló, I. \& Lozano, J.M. (2011). Searching for New Forms of Legitimacy Through Corporate Responsibility Rhetoric. Journal of Business Ethics, 100(1), 11-29.

Chen, H., Griffith, D. \& Hu, M. (2006). The influence of liability of foreignness on market entry strategies: An illustration of market entry in China. International Marketing Review, 23(6), 636-649.

Chin, W. (1998). The Partial Least Square Approach to Structural Equation Modeling. En G.A. Marcoulides (Ed), Modern Methods for Business Research (pp. 295-336). Mahwah, NJ: Lawrence Erlbaum Associates, Publisher.

De Bakker, F. G., Groenewegen, P. \& Den Hond, F. (2005). A bibliometric analysis of 30 years of research and theory on Corporate Social Responsibility and Corporate Social Performance. Business \& Society, 44(3), 283-317.

De Grosbois, D. (2016). Corporate social responsibility reporting in the cruise tourism industry: A performance evaluation using a new institutional theory based model. Journal of Sustainable Tourism, 24(2), 245-269. doi:10.1080/09669582.2015.1076827

Deephouse, D.L. (1996). Does isomorphism legitimate?. Academy of Management Journal, 39(4), 1024-1039.

Dias Alperstedt, G., Martignago, G., \& Gonçalves Silveira Fiates, G. (2006). O processo de adaptação estratégica em uma instituição de ensino superior sob a ótica da Teoria Institucional. Revista de Ciências da Administração, 8(15), 114-137. 
Díez Martín, F., Blanco González, A. \& Prado Román, C. (2010). Medición de la legitimidad organizativa: El caso de las Sociedades de Garantía Recíproca. Cuadernos de Economía y Dirección de la Empresa, 13(43), 115-143.

Dimaggio, P. J. \& Powell, W. W. (1983). The iron cage revisited: Institutional isomorphism and collective rationality in organizational fields. American Sociological Review, 48(2), 147-160.

DiMaggio, P. J. \& Powell, W.W. (1991). The Iron Cage Revisited: Institutional Isomorphism and Collective Rationality in Organization Fields. En W. W. Powell and P. J. DiMaggio (Eds.), The New Institutionalism in Organizational Analysis (pp. 63-82). Chicago, IL: University of Chicago Press.

Dobbin, F., Meyer, J.W., Scott, R. \& Sutton, J.R. (1993). Equal Opportunity Law and the Construction of Internal Labor Markets. American Journal of Sociology, 99, 396-427.

Egels-Zandén, N. \& Kallifatides, M. (2006). The corporate social performance dilemma: Organizing for goal duality in low-income African markets. En Wayne Visser, Malcolm McIntosh \& Charlotte Middleton (Eds.) Corporate citizenship in Africa: Lessons from the past; paths to the future (pp. 82-92). Sheffield: Greenleaf Publishing.

Egels-Zandén, N. \& Wahlqvist, E. (2007). Post-partnership strategies for Refining corporate responsibility: The business social compliance initiative. Journal of Business Ethics, 70(2), 175-189.

Falk, R.F. \& Miller; N.B. (1992). A Primer for Soft Modeling. Akron, Ohio: The University of Akron Press.

Fernández, M.L. (2001). Un análisis institucional del contexto y su incidencia en el proceso de cambio en la gestión de los recursos humanos. Tres estudios de casos. Tesis Doctoral, Universidad de Cádiz. Facultad de Ciencias Económicas y Empresariales.

Fornell, C. \& Lacker, D. (1981). Evaluating structural equation models with unobservable variables and measurement error: algebra and stadistic. Journal of Marketing Research, 28(Febrero), 39-50.

Galaskiewicz, J. (1985). Interorganizational relations. Annual Review of Sociology, 11, 281-304.

Gallardo-Vazquez, D., \& Sanchez-Hernandez, I. (2012). Information on corporate social responsibility and SME's environmental responsiveness: A regional study. Economics and Sociology, 5(2), 103-115.

Gerde, V.W. \& Wokutch, R.E. (1998). 25 years and going strong: A content analysis of the first 25 years of the social issues in management division proceedings. Business and Society, $37(4), 414-446$. 
Godos, J. \& Fernández, R. (2011). ¿Cómo se percibe la dirección socialmente responsable por parte de los altos directivos de empresas en España?. Universia Business Review, 29, 3249.

Golant, B.D. \& Sillince, J.A.A. (2007). The constitution of organizational legitimacy: A narrative perspective. Organization Studies, 28, 1149-1167.

Hunt, C. S. \& Aldrich, H.E. (1996). Why even Rodney Dangerfield has a home page: Legitimizing the world wide web as a medium for commercial endeavours. Congreso Anual de la Academy of Management, Cincinnati, $\mathrm{OH}$.

Jennings, P.D. \& Zandbergen, P.A. (1995). Ecologically sustainable organizations: An institutional approach. Academy of Management Review, 20(4), 1015-1052.

Kostova, T. \& Roth, K. (2002). Adoption o fan organizational practice by subsidiaries of multinational corporations: Institutional and relational effects. Academy of Management Journal, (45), 215-243.

Kostova, T. \& Zaheer, S. (1999). Organizational legitimacy under conditions of complexity: The case of the multinational enterprise. Academy of Management Review, 24, 64-81.

Kumar, N., Stern, L. \& Anderson, J. (1993). Conducting interorganizational research using key informants. Academy of Management Journal, 36, 1633-1651.

Lavandoski, J., Vargas-Sánchez, A. Pinto, P., \& Silva, J. A. (2016) Causes and effects of wine tourism development in organizational context: The case of Alentejo, Portugal. Tourism and Hospitality Research, 1, 1-16.

Llamas-Sanchez, R., Garcia-Morales, V., \& Martin-Tapia, I. (2013). Factors affecting institutional change: A study of the adoption of Local Agenda 21 in Spain. Journal of Organizational Change Management, 26(6), 1045-1070. doi:10.1108/JOCM-03-2012-0037

Lockett, A.; Moon, J. \& Visser, W. (2006). Corporate social responsibility in management research: Focus, nature, salience and sources of influence. Journal of Management Studies, 43(1), 115-136.

Lopes, E. R. [coord.] (2010). A constelação do turismo na economia portuguesa, Mirandela: Edições Jornal Sol.

Lozano, F. J. \& Boni, A. (2002). The impact of the multinational in the development: An ethical challenge. Journal of Business Ethics, 39(1-2), 169-178.

Meyer, J. W. \& Scott W. R. (1983). The organization of the societal sectors. En John W. Meyer e W. Richard Scott (Eds.) Organizational Environments: Ritual and Rationality (pp. 100-121), Beverly Hills, CA: Sage.

Meyer, J. \& Rowan, B. (1977). Institutionalized organizations: Formal structure as mythand ceremony. The American Journal of Sociology, 83(2), 340-363.

Revista de Gestão e Secretariado-GeSec, São Paulo, v. 7, n. 2, p 191-212,mai./ago. 2016. 
Moyano-Fuentes, J.E. (2001). Nuevos modelos de competencia en ecología organizativa: análisis empírico en la industria de extracción de aceite de oliva en la provincia de Jaén, 1944-1998. Tesis Doctoral. Universidad de Jaén. Departamento de Administración de Empresas, Contabilidad y Sociología.

Murillo Vargas, G., González Campo, C.H. \& Rodriguez Orejuela, H.A. (2010) Corporate social responsibility in the context of institutional and organizational change in the Colombian financial sector, Revista AD-Minister, 17(Julio-Diciembre), 59-85.

Nunnally, J.C. (1978). Psychometric theory (2 ed.). New York: McGraw-Hill.

Oliver, C.H. (1991). Strategic Responses to Institutional Processes. Academy of Management Review, 16(1), 145-179.

Palazzo, G. \& Scherer, A.G. (2006). Corporate legitimacy as deliberation: A communicative Framework. Journal of Business Ethics, 66(1), 71-88.

Perdomo, J. \& Escobar, A. (2011). La investigación en RSE : una revisión desde el management. Cuadernos de Administración, 24(43), 193-219.

Pérez López, C. (2005). Métodos estadísticos avanzados con Spss. Madrid: Thomson.

Piazza, J. (2002). Going global - Unions and globalization in the United States, Sweden, and Germany. Lanham, MD: Lexington Books.

Restrepo, M.C. \& Rosero, X. X. (2002). Teoría Institucional y proceso de internacionalización de las empresas colombianas. Estudios Gerenciales, 18(84), 103-123.

Riquel Ligero, F. J. (2010) Análisis institucional de las prácticas de gestión ambiental de los campos de golf andaluces. Tesis de doctorado. Universidad de Huelva. Facultad de Ciencias Empresariales.

Rivera, J. (2004). Institutional pressures and voluntary environmental behavior in developing countries: Evidence from the costa rican hotel industry. Society and Natural Resources, 17(9), 779-797.

Roldán, J. (2000). Sistemas de información ejecutivos EIS. Génesis, implantación y repercusiones organizativas. Tesis Doctoral. Universidad de Sevilla.

Rowley, T. \& Berman, S. (2000). A brand new brand of corporate social performance. Business \& Society, 39, 397-418.

Ruef, M. \& Scott, R. (1998). A multidimensional model of organizational legitimacy: Hospital survival in changing institutional environments. Administrative Science Quarterly, 43(4), 877-904.

Sarabia Sánchez, F.J., et al. (1999). Metodología para la investigación en marketing y dirección de empresas. Madrid: Pirámide.

Revista de Gestão e Secretariado-GeSec, São Paulo, v. 7, n. 2, p 191-212, mai./ago. 2016. 
Scherer, A. \& Palazzo, G. (2007). Toward a Political Conception of Corporate Responsibility: Business and Society Seen from a Habermasian Perspective. Academy of Management Review, 32(4), 1096-1120.

Scott, W. R. (1995). Institutions and organizations. Thousand Oaks, CA: Sage.

Shah, K. U., \& Rivera, J. E. (2007). Export processing zones and corporate environmental performance in emerging economies: The case of the oil, gas, and chemical sectors of trinidad and tobago. Policy Sciences, 40(4), 265-285.

Shah, K. U., \& Rivera, J. E. (2013). Do industry associations influence corporate environmentalism in developing countries? evidence from trinidad and tobago. Policy Sciences, 46(1), 39-62.

Suchman, M. (1995). Managing legitimacy: Strategic and institutional approaches. Academy of Management Review, 20(3), 571-610.

Vargas-Sánchez, A., \& Riquel-Ligero, F.J. (2016). Institutional Pressures, Environmental Responsibility and Social Legitimacy: Longitudinal Analysis Applied to Golf Tourism. In Matias, Á. Nijkamp, P., \& Romão, J. (Eds.) Impact Assessment in Tourism Economics (pp.173-187). Switzerland: Springer International Publishing.

Vargas-Sánchez, A., \& Riquel-Ligero, F. (2012) Influence of the institutional context on the performance of golf courses, considering the natural environment. Environmental Engineering and Management Journal, 11(11), 2001-2012.

Verdu Jover, A.J. (2002). Relación entre flexibilidad y desempeño organizativo: una aproximación desde la perspectiva de la gestión de la calidad total. Alicante: Universidad Miguel Hernández.

Vitell, S. J.; Ramos, E. \& Nishihara, C.M. (2010). The Role of Ethics and Social Responsibility in Organizational Success: A Spanish Perspective. Journal of Business Ethics, 91(4), 467-483.

Voss, G., Cable, D. \& Giraud, Z. (2000). Linking organizacional values to relationships with external constituents: A study of nonprofit professional tetares. Organization Science, 11(3), 330-347.

Walsh, J., Weber K. \& Margolis J. (2003). Social Issues and Management: Our Lost Cause Found. Journal of Management, 29, 859-881.

Wang, C.H. (2005). Constructing multivariate process capability indices for short-run production. Int. J. Adv. Manuf. Technol, 26, 1306-1311.

Wubneh, M.A. (1987). Multivariate analysis of socio-economic charasteristics of urban areas in Ethiopia. Afr. Urban Quaterly, 2, 425-433.

Revista de Gestão e Secretariado-GeSec, São Paulo, v. 7, n. 2, p 191-212,mai./ago. 2016. 
Zapata, M. J., \& Hall, C. M. (2012). Public-private collaboration in the tourism sector: Balancing legitimacy and effectiveness in local tourism partnerships. The Spanish case. Journal of Policy Research in Tourism, Leisure and Events, 4(1), 61-83.

Zhu, Q., Sarkis, J., \& Lai, K. -. (2011). An institutional theoretic investigation on the links between internationalization of chinese manufacturers and their environmental supply chain management. Resources, Conservation and Recycling, 55(6), 623-630. 